\title{
An e-survey exploring adherence to the Mediterranean Diet and associated barriers to healthy eating among British adults of retirement age
}

\author{
J. Lara, L. A. McCrum and J. C. Mathers \\ Human Nutrition Research Centre, Institute for Ageing and Health; Newcastle University. Biomedical Research \\ Building, Campus for Ageing and Vitality. Newcastle Upon Tyne, NE4 5PL, UK
}

Strong evidence suggests that adherence to a Mediterranean Diet (MD) is associated with lower risk of a wide range of ageing-related diseases including cardiovascular disease, cancer and Alzheimer's disease. The MD describes a dietary pattern which is characterised by higher intakes of vegetables, fruits, legumes, cereals, fish and a moderate intake of red wine during meals. This study is part of LiveWell, a research programme to develop lifestyle-based interventions to improve healthy ageing at the peri-retirement period. The aims of the present study were to assess adherence to a MD among a group of older British adults and to identify associated barriers to healthy eating. Eighty men and 120 women completed an online survey on eating habits in the UK. Adherence to MD was assessed using the 14-item PREDIMED score (MDPS) ${ }^{(1)}$. Participants were also asked to identify perceived barriers to healthy eating (PBHE) from a list of 22 barriers previously published ${ }^{(2)}$. Two-step cluster analysis was used to identify groups of barriers, and their association with MD was assessed using analysis of variance.

Participants of this survey were 61 (SD 7) years of age, and overweight (BMI 26 SD $4 \mathrm{~kg} / \mathrm{m} 2$ ) adults. Overall adherence to MD as measured by MDPS was 5.6 (SD 2), with 31\% of participants scoring $\geq 7$. Participants reported a mean of 4 (SD 2; range 1 to 11 ) PHBE. The number of PBHE was positively associated with BMI $(r=0 \cdot 28, p<0.001)$, and inversely associated with age $(r=-0 \cdot 19$; $p=0.006)$, and with MDPS $(r=-0.31 ; p<0.001)$. Two-step cluster analysis produced three clusters; cluster- 1 associated with busy lifestyle time, irregular working hours, or the belief that healthy eating involves lengthy preparation; cluster-2 associated with lack of willpower and finding hard to give-up liked foods; and cluster-3 in which no prominent PBHE was identified. On average, participants in cluster-1 were younger (57 years), heavier $\left(28 \mathrm{~kg} / \mathrm{m}^{2}\right)$, scored lower on MDPS (mean $\left.=4.7\right)$ and reported more PBHE (mean = 7). Participants in cluster-3 were leaner $\left(25.5 \mathrm{~kg} / \mathrm{m}^{2}\right)$, scored higher on MDPS $($ mean $=6 \cdot 2)$, and reported the lowest number of PBHE (mean = 2).

In conclusion, even among participants with relatively moderate adherence to the MD, those reporting more PBHE had lower MDPS and higher BMI. These findings may be useful in developing dietary interventions promoting the MD within the UK.

1. Schröder H, Fitó M, Estruch R, et al. (2011) J Nutr 141, 1140-1145.

2. Kearney JM, McElhone S (1999) Br J Nutr 81, Suppl 2, S133-7.

The LiveWell project is funded by the Lifelong Health and Wellbeing Cross-Council Programme initiative in partnership with the UK Health Department: The LLHW Funding Partners are: Biotechnology and Biological Sciences Research Council, Engineering and Physical Sciences Research Council, Economic and Social Research Council, Medical Research Council, Chief Scientist Office of the Scottish Government Health Directorates, National Institute for Health Research /The Department of Health, The Health and Social Care Research \& Development of the Public Health Agency (Northern Ireland), and Wales Office of Research and Development for Health and Social Care, Welsh Assembly Government. 